\title{
Effective discrimination of chiral molecules in a cavity
}

\author{
Yi-HaO KAnG ${ }^{1,2}$, Zhi-Cheng Shi ${ }^{1,2}$, JiE SONG ${ }^{3}$, AND YAN XIA ${ }^{1,2}$ \\ ${ }^{1}$ Fujian Key Laboratory of Quantum Information and Quantum Optics (Fuzhou University), Fuzhou 350116, China \\ ${ }^{2}$ Department of Physics, Fuzhou University, Fuzhou 350116, China \\ ${ }^{3}$ Department of Physics, Harbin Institute of Technology, Harbin 150001, China \\ *Yan Xia: xia-208@163.com
}

Compiled August 10, 2020

\begin{abstract}
We present a scheme to realize precise discrimination of chiral molecules in a cavity. Assisted by additional laser pulses, cavity fields can evolve to different coherence states with contrary-sign displacements according to the handedness of molecules. Consequently, the handedness of molecules can be read out with homodyne measurement on the cavity, and the successful probability is nearly unity without very strong cavity fields. Numerical results show that the scheme is insensitive to errors, noise and decoherence. Therefore, the scheme may provide helpful perspectives for accurate discrimination of chiral molecules. ๑ 2020 Optical Society of America
\end{abstract}

http://dx.doi.org/10.1364/ao.XX.XXXXXX

Chiral molecules, composed of enantiomers being mirror images of each other, have shown many interesting applications in chemistry, biotechnologies, and pharmaceutics [1-3]. Although a pair of enantiomers share many physical and chemical properties, their own special properties originated from the handedness show divergent behaviors in chemical and biological reactions. Therefore, it is necessary to detect and separate racemic mixtures of enantiomers. However, discrimination of chiral molecules is usually a difficult task in chemistry [4], since chemical techniques, such as crystallization, derivatization, kinetic resolution, and chiral chromatography [5] are usually time-consuming and expensive.

Fortunately, researches [6-11] have shown that electricdipole interactions between chiral molecules and linearly polarized light have potential to realize enantioselectivity as some of transition dipole moments of left $(L)$ and right $(R)$ handed enantiomers have different signs. Thus, by using filed-molecule couplings, it is possible to rapidly detect handedness of molecules based on the difference of evolutions. From this point, schemes for the discrimination of chiral molecules with field-molecule couplings are proposed by using techniques like adiabatic passage [12-14] and flat resonant pulses [15]. Interestingly, recent schemes [16-18] using the technique "shortcut to adiabaticity" [19-22] further make trade-off between the evolution speed and robustness to errors, which make discrimination of chiral molecules more effective. However, in these previous schemes [13-18], discrimination of enantiomers relies on the population difference, and requires higher molecule levels and coher- ence superposition in the process. As a result, decoherence of molecules, e.g., energy relaxation and dephasing may cause significant population errors, and pursuing an acceptable successful probability would impose high requirement on the coherence time of molecules. On the other hand, because population of a molecule state can only ranges between 0 and 1 , and is proportional to the successful probability, small deviations of pulse areas may also significantly reduce successful probabilities of the discrimination. Therefore, if the information of handedness can be stored and read out in some stable ways instead of using populations of molecules, the discrimination of chiral molecules can be more accurate in the presence of experimental imperfections.

In this letter, we propose an effective scheme to discriminate chiral molecules. Apart from external driving pulses applied to molecules, we also consider couplings of molecules with the quantized field in a cavity. Instead of the population difference, information of handedness of enantiomers in the scheme can be perfectly acquired from coherence states of cavity fields with contrary-sign displacements by using homodyne measurement [25-28]. The error rates of the scheme are described by complementary error functions of the difference of the displacements. When we set proper final displacements of coherence state, the successful probabilities are insensitive to deviations of the displacements caused by systematic errors and random noise. Thus, compared with schemes using population difference, where successful probabilities are proportional to the population difference, the scheme can be more robust against errors and noises. Moreover, in the scheme, molecules are almost restricted in their ground states, thus robustness against energy relaxation and dephasing of molecules can be enhanced. Numerical results indicates that the requirement of coherence time in the scheme is much looser than the previous schemes $[17,18]$ when energy relaxation, dephasing and cavity decay are all taken into account. Therefore, the scheme may provide a feasible way to discriminate chiral molecules.

Let us now describe the physical model in detail. As shown in Fig. 1, we consider two enantiomers of chiral molecules with $L$ - and $R$-handedness. Both of $L$ - and $R$-handed molecules have three discrete energy states, denoted by $\left\{|1\rangle_{L},|2\rangle_{L},|3\rangle_{L}\right\}$ and $\left\{|1\rangle_{R},|2\rangle_{R},|3\rangle_{R}\right\}$, respectively. Due to the difference of handedness, the transition-dipole moment $\vec{\mu}_{13}$ is in different directions for $L$ - and $R$-handed molecules, while the transition-dipole moment $\vec{\mu}_{12}\left(\vec{\mu}_{23}\right)$ is in the same direction for $L$ - and $R$-handed 


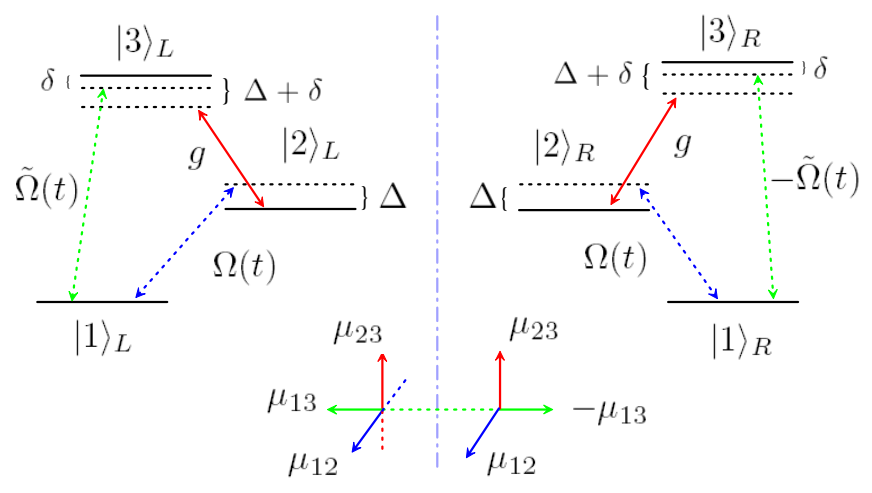

Fig. 1. Comparison of the coupling schemes between three discrete energy states in molecules with left $(L)$ and right $(R)$ handedness.

molecules [16-18]. We assume the transition $|1\rangle \leftrightarrow|2\rangle$ is driven by a classical field $\vec{E}(t)=\varepsilon(t) \cos (\omega t) \vec{e}_{12}$ with frequency $\omega$, amplitude $\varepsilon(t)$ and polarization direction $\vec{e}_{12}$. Assuming that the transition frequency of $|1\rangle \leftrightarrow|2\rangle$ is $\omega_{12}=\omega-\Delta$, the Rabi frequency and the (blue) detuning of the transition should be $\Omega(t)=\varepsilon(t) \vec{\mu}_{12} \cdot \vec{e}_{12}$ and $\Delta$. In addition, the transition $|2\rangle \leftrightarrow|3\rangle$ couples with a quantized cavity mode with frequency $v$ in a cavity with coupling strength $g=\sqrt{\frac{v}{2 \epsilon V}} \vec{\mu}_{23} \cdot \vec{e}_{23}$, with $\vec{e}_{23}$ being the unit polarization vector for cavity mode, $\epsilon$ being dielectric constant of the dielectric of the cavity, and $V$ being the volume of the cavity. For the transition frequency $\omega_{23}=v+\Delta+\delta$, the (red) detuning of the coupling is $\Delta+\delta$. Moreover, the transition $|1\rangle \leftrightarrow|3\rangle$ is also driven by a classical field $\overrightarrow{\tilde{E}}(t)=$ $\tilde{\varepsilon}(t) \cos (\tilde{\omega} t) \vec{e}_{13}$ with frequency $\tilde{\omega}$, amplitude $\tilde{\varepsilon}(t)$ and polarization direction $\vec{e}_{13}$. For the transition frequency $\omega_{13}=\tilde{\omega}+\delta$, the (red) detuning of the transition is $\delta$. However, due to different handedness, the Rabi frequency is $\tilde{\Omega}(t)=\tilde{\varepsilon}(t) \vec{\mu}_{13} \cdot \vec{e}_{13}$ for $L-$ handed molecule but becomes $-\tilde{\Omega}(t)$ for $R$-hand molecule. In the rotating-wave-approximation, the total Hamiltonian of an enantiomer reads

$$
\begin{aligned}
H(t) & =\Omega(t) e^{i \Delta t}|1\rangle\left\langle 2\left|+g a e^{i(\Delta+\delta) t}\right| 3\right\rangle\langle 2| \\
& \pm \tilde{\Omega}(t) e^{-i \delta t}|1\rangle\langle 3|+\text { H.c., }
\end{aligned}
$$

with $a$ being the annihilate operator of the cavity mode and $\pm \tilde{\Omega}(t)$ for $L$ - and $R$-handed molecules, respectively. We consider the conditions $\tilde{\Omega}(t) \sim g^{2} / \Delta \ll \delta, \Omega(t) \sim g \ll \Delta$ and $\delta \ll \Delta$. The effective Hamiltonian can be derived via secondorder perturbation theory [23] as

$$
\begin{aligned}
& H_{e}(t)=H_{1}(t)+H_{2}(t)+H_{3}(t), \\
& H_{1}(t)=-\frac{\Omega^{2}(t) g^{2}}{\Delta^{2} \delta} a^{\dagger} a|1\rangle\langle 1|, \\
& H_{2}(t)=\left[\frac{\Omega^{2}(t)}{\Delta}-\frac{\tilde{\Omega}^{2}(t)}{\delta}\right]|1\rangle\langle 1|, \\
& H_{3}(t)=\mp \frac{\Omega(t) \tilde{\Omega}(t) g}{\Delta \delta}\left(a^{\dagger}+a\right)|1\rangle\langle 1| .
\end{aligned}
$$

Assuming the considered enantiomer is initial in the lowest state $|1\rangle, H_{2}(t)$ only leads a global phase, thus can be omitted. In the rotation frame of $\mathcal{R}=\exp \left[-i \int_{0}^{t} H_{1}\left(t^{\prime}\right) d t^{\prime}\right]$, the effective Hamiltonian can be simplified as

$$
\tilde{H}_{e}(t)=\mp \Omega_{e}(t)\left[e^{-i \varphi(t)} a^{\dagger}+e^{i \varphi(t)} a\right]|1\rangle\langle 1|,
$$

with $\Omega_{\mathcal{e}}(t)=\Omega(t) \tilde{\Omega}(t) g / \Delta \delta, \varphi(t)=\int_{0}^{t} d t^{\prime} \Omega^{2}\left(t^{\prime}\right) g^{2} / \Delta^{2} \delta$, and $\mp \Omega_{e}(t)$ for $L$ - and $R$-handed molecules, respectively.

Assuming the cavity is initial in the vacuum state, evolution (density operator) of the system can be calculated as [24]

$$
\begin{aligned}
& \tilde{\rho}_{e}(t)=| \pm \tilde{\alpha}(t)\rangle_{c}\langle \pm \tilde{\alpha}(t)|\otimes| 1\rangle_{e}\langle 1|, \\
& \tilde{\alpha}(t)=\int_{0}^{t} i \Omega_{e}\left(t^{\prime}\right) e^{-i \varphi\left(t^{\prime}\right)} d t^{\prime},
\end{aligned}
$$

with subscript $c$ and $e$ corresponding to the states of cavity and enantiomer. In addition, $|\tilde{\alpha}(t)\rangle_{c}$ is a coherence state of cavity. Therefore, back to the original frame, the evolution at final time $t=T$ can be described by

$$
\rho_{e}(T)=| \pm \alpha(T)\rangle_{c}\langle \pm \alpha(T)|\otimes| 1\rangle_{e}\langle 1|,
$$

with $\alpha(T)=\tilde{\alpha}(T) e^{i \varphi(T)}$. According to the result, for $L$-handed molecules, the cavity is finally in state $|\alpha(T)\rangle$, while for $R$ handed molecules, the cavity is finally in state $|-\alpha(T)\rangle$. When $|\alpha(T)| \gg 1,|\langle-\alpha(T) \mid \alpha(T)\rangle| \sim 0$, the difference of cavity can be easily discriminated by a $X_{\phi}$-quadrature homodyne measurement [25-28] with a probing mode in coherence state $|z\rangle$ with $\phi=\arg (z)=\arg [\alpha(T)]$. This is equivalent to the eigenprojection $|x, \phi\rangle\langle x, \phi|$ of operator $X_{\phi}=e^{i \phi} a^{\dagger}+e^{-i \phi} a$. Considering the probability distribution $f_{ \pm}(x, \phi)=|\langle x, \phi \mid \pm \alpha(T)\rangle|^{2}=$ $(2 / \pi)^{1 / 2} \exp \left[-2(x \pm|\alpha|)^{2}\right][25,28]$, if we consider result of homodyne measurement result with $x>0(x<0)$ for the cavity in state $|\alpha(T)\rangle(|-\alpha(T)\rangle)$, the error probability is $P_{e}=$ $\int_{0}^{+\infty} f_{-}(x, \phi) d x=\operatorname{erfc}[\sqrt{2}|\alpha(T)|] / 2$, which becomes less than $3.16712 \times 10^{-5}$ when $|\alpha(T)|>2$. Therefore, the scheme can give near deterministic discrimination of chiral molecules.

We now using numerical simulations to show the performance of the scheme. Using the coupling strength $g$ as a reference value, we select control functions as $\tilde{\Omega}(t)=$ $A_{1} g \sin (\pi t / T)$ and $\Omega(t)=A_{2} g \sin (\pi t / T)$, with two dimensionless parameters $A_{1}$ and $A_{2}$. In this way, we obtain $\alpha(T)=$ $A_{1} A_{2} g^{3} T \exp \{i[\pi(1 / 2-\mu)+\varphi(T)]\} \sin (\pi \mu) /(2 \pi \mu \Delta \delta), \mu=$ $A_{2}^{2} g^{4} T /\left(4 \pi \Delta^{2} \delta\right)$, and $\varphi(T)=A_{2}^{2} g^{4} T /\left(2 \Delta^{2} \delta\right)$. Accordingly, the angle should be $\phi=\pi(1 / 2-\mu)+\varphi(T)$ in the $X_{\phi}$-quadrature homodyne measurement. In addition, $A_{1} \geq A_{2} g /[\Delta \sin (\pi \mu)]$ should be satisfied to make $|\alpha(T)| \geq 2$. To examine the relations between parameters $A_{1}, A_{2}$ and $T$, we first set $\Delta=20 \mathrm{~g}$ and $\delta=20 g^{2} / \Delta$ for conditions $\Delta \gg g$ and $\delta \gg g^{2} / \Delta$, and we plot $A_{1}=A_{2} g /[\Delta \sin (\pi \mu)]$ versus $A_{2}$ and $T$ in Fig. 2(a). As shown

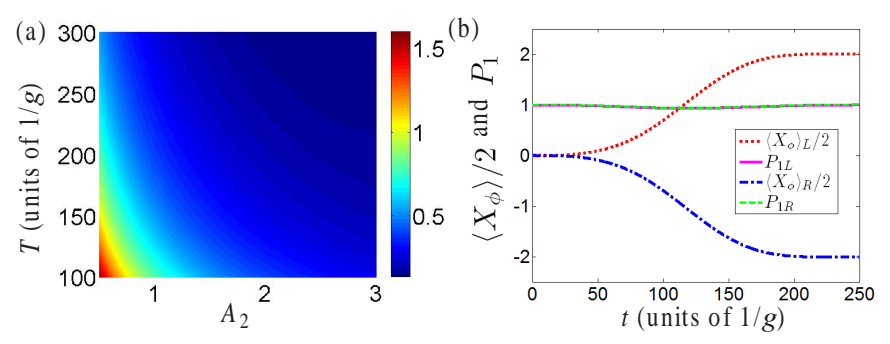

Fig. 2. (a) $A_{1}=A_{2} g /[\Delta \sin (\pi \mu)]$ versus $A_{2}$ and $T$. (b) $\left\langle X_{\phi}\right\rangle / 2$ and $P_{1}$ versus $t$ with $L$-handed molecule (red-dotted line: $\left\langle X_{\phi}\right\rangle_{L} / 2$; magenta-solid line: $\left.P_{1 L}\right)$ and $R$-handed molecule (blue-dashed-and-dotted line: $\left\langle X_{\phi}\right\rangle_{R} / 2$; green-dashed line: $\left.P_{1 R}\right)$.

in Fig. 2(a), when $\Delta \gg g$ and $\delta \gg g^{2} / \Delta, A_{1}$ decreases when $A_{2}$ and $T$ increase. Since $\tilde{\Omega}(t) \sim g^{2} / \Delta$ should be satisfied, we consider a proper value $A_{1}=0.15$ at point $\left(A_{2}, T\right)=(2.5,250 / g)$. 
With the selections of parameters, we plot the variation of the average value $\left\langle X_{\phi}\right\rangle / 2=\operatorname{Tr}\left[X_{\phi} \rho(t)\right] / 2$ versus $t$ in Fig. 2(b). According to Fig. 2(b), $\left\langle X_{\phi}\right\rangle / 2$ varies from 0 to \pm 2.068 when the considered molecules are $L$ - and $R$-handed, respectively. Noticing that $\left\langle X_{\phi}\right\rangle=2|\alpha(T)|$ is satisfied when $\phi=\arg [\alpha(T)]$, the numerical result accords with the theory analysis based on the effective Hamiltonian. Considering a reported coupling strength $g=10 \mathrm{MHz}$ [29] for molecules in cavity, the total interaction time required is $T=25 \mu \mathrm{s}$. Thus, $L$ - and $R$-handed molecules can be fast discriminated via homodyne measurement with error rates below $3.16712 \times 10^{-5}$. Moreover, we also examine the population $P_{1}$ of state $|1\rangle$ in the evolution. Seen from the magenta-solid line and the green-dashed line, populations of both $L$ - and $R$-handed molecules almost keep at 1 during the evolution. This also proves the validity of the theory analysis.

We now demonstrate the performance of the scheme in the presence of experimental imperfections. First, due to operational and instrumental imperfection, there may exist systematic errors in the Rabi frequencies and coupling strengths. For the scheme, we assume systematic errors as $\tilde{\Omega}(t) \rightarrow(1+$ $\left.\eta_{1}\right) \tilde{\Omega}(t), \Omega(t) \rightarrow\left(1+\eta_{2}\right) \Omega(t)$ and $g \rightarrow\left(1+\eta_{3}\right) g$ with $\eta_{j}$ $(j=1,2,3)$ being the faulty coefficient. We plot $D(X / 2)=$ $\left(\left\langle X_{\phi}\right\rangle_{L}-\left\langle X_{\phi}\right\rangle_{R}\right) / 2$ versus $\eta_{j}$ in Fig. 3(a). In Fig. 3(a), with the increase of $\eta_{1}$ from 0 to $0.1, D(X / 2)$ also increases. Theoretically, according to the effective Hamiltonian, systematic error of $\tilde{\Omega}(t)$ only influences the displacement $\alpha(T)$, consequently $|\alpha(T)|$ increases when $\eta_{1}>0$. However, systematic errors of $\Omega(t)$ and $g$ influence both displacement $\alpha(T)$ and angle $\varphi(T)$, thus both increase and decrease of $\eta_{2}$ and $\eta_{3}$ may reduce $D(X / 2)$ due to the mismatching of angles. According to the results, when $\eta_{j} \in[-0.1,0.1], D(X / 2)$ remains higher than 3.77. Therefore, the error rate of homodyne measurement is still below $8.162 \times 10^{-5}$. In addition, we examine the systematic errors of detunings as $\Delta \rightarrow\left(1+\eta_{1}^{\prime}\right) \Delta$ and $\delta \rightarrow\left(1+\eta_{2}^{\prime}\right) \delta . D(X / 2)$ versus $\eta_{1}^{\prime}$ and $\eta_{2}^{\prime}$ is shown in Fig. 3(b). The result indicates that $D(X / 2)$ tends to decrease with the increase of $\eta_{1}^{\prime}$ and $\eta_{2}^{\prime}$ when $\eta_{1}^{\prime}, \eta_{2}^{\prime} \in[-0.1,0.1]$. This is because $\Omega_{e}(t)$ decreases when $\Delta$ and $\delta$ increase. The worst case appears at $\left(\eta_{1}^{\prime}, \eta_{2}^{\prime}\right)=(0.1,0.1)$, which gives $D(X / 2)=3.601$. In this case, the error rate of homodyne measurement is only $P_{e}=1.5911 \times 10^{-4}$. Therefore, the scheme provides nice fault-tolerance for the discrimination of chiral molecules.

Apart from systematic errors, random noise due to fluctuation of experimental environment also disturb evolutions of physical systems. As the additive white Gaussian noise (AWGN) is a nice model to describe random noise, we here consider the influence of AWGN in the pulses. Rabi frequency $Y(t) \in\{\tilde{\Omega}(t), \Omega(t)\}$ under the influence of the AWGN is $Y_{N}(t)=Y(t)+\operatorname{awgn}\left(Y(t), R_{N}\right)$, where $\operatorname{awgn}\left(Y(t), R_{N}\right)$ is a function generating AWGN with signal-to-noise ratio (SNR) $R_{N}$ for pulse, and $Y_{N}(t)$ is the pulse involves AWGN. To estimate the influence of AWGN in average, we perform fifty simulations with SNR $R_{N}=10$, and the results are shown in Fig. 3(c). According to the results, the worst result in the fifty simulations is $D(X / 2)=3.215$, corresponding to error rate $P_{e}=6.5222 \times 10^{-4}$. Therefore, the scheme also holds good robustness against random noise.

Finally, let us consider the influence of decoherence to the operation. For molecules, the energy relaxation and dephasing are two disturbing factor destroying the coherence. On the other hand, the cavity decay weakens intensity of the cavity field, which may also reduce the successful probability of homodyne measurement. In the presence of these decoherence factors, the
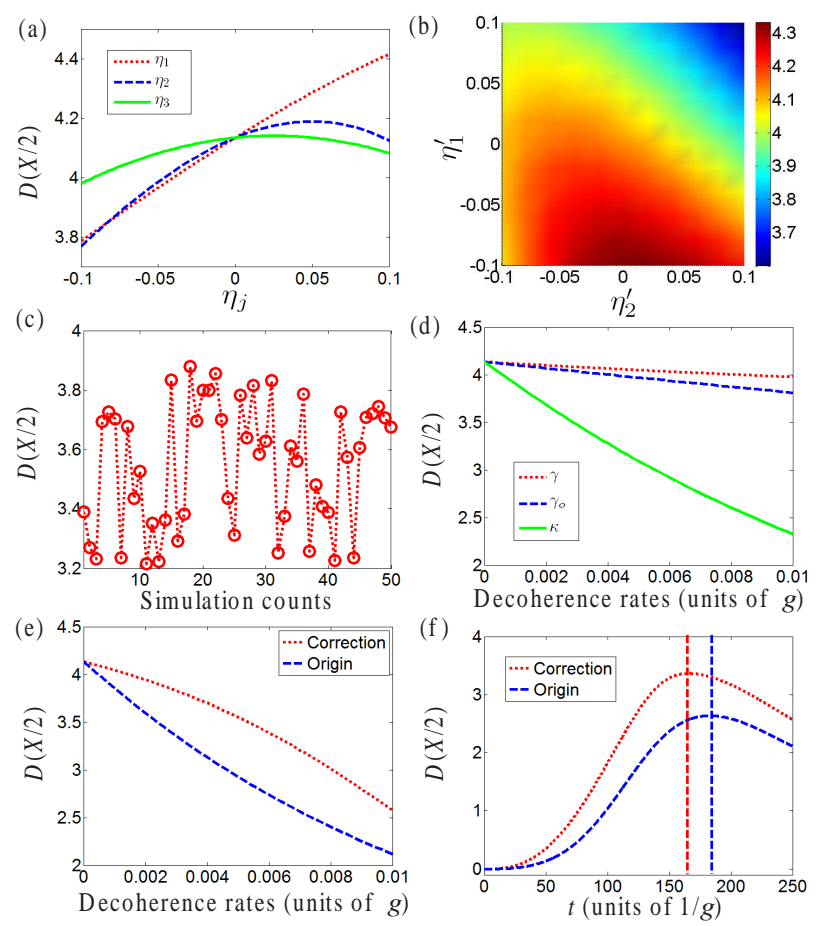

Fig. 3. (a) $D(X / 2)$ versus $\eta_{j}$. (b) $D(X / 2)$ versus $\eta_{1}^{\prime}$ and $\eta_{2}^{\prime}$. (c) $D(X / 2)$ versus simulation counts under AWGN with SNR $R_{N}=10$. (d) $D(X / 2)$ versus decoherence rates $\gamma, \gamma_{\phi}$ and $\kappa$. (e) $D(X / 2)$ versus decoherence rate $\left(\gamma=\gamma_{\phi}=\kappa\right)$ with corrected pulses and original pules. (f) $D(X / 2)$ versus $t$ with corrected pulses and original pules $\left(\gamma=\gamma_{\phi}=\kappa=0.01 g\right)$.

evolution is governed by a master equation as

$$
\begin{aligned}
\dot{\rho}(t) & =-i[H(t), \rho(t)]+\frac{\kappa}{2} \mathcal{L}[a] \rho(t) \\
& +\sum_{\iota=1}^{2} \sum_{\iota^{\prime}=\iota+1}^{3}\left\{\frac{\gamma}{2} \mathcal{L}\left[\sigma_{u^{\prime}}^{-}\right] \rho(t)+\frac{\gamma_{\phi}}{2} \mathcal{L}\left[\sigma_{\iota^{\prime}}^{z}\right] \rho(t)\right\}
\end{aligned}
$$

with $\mathcal{L}[o] \rho(t)=2 o \rho(t) o^{\dagger}-o^{\dagger} o \rho(t)-\rho(t) o^{\dagger} o$, cavity decay rate $\kappa$, energy relaxation rate $\gamma$, dephasing rate $\gamma_{\phi}, \sigma_{u^{\prime}}^{-}=|\iota\rangle\left\langle\iota^{\prime}\right|$, and $\sigma_{\iota^{\prime}}^{z}=\left(\left|\iota^{\prime}\right\rangle\left\langle\iota^{\prime}|-| \iota\right\rangle\langle\iota|\right) / 2$. We plot $D(X / 2)$ versus different decoherence rates in Fig. 3(d). The results show that when $\kappa=0.01 g, \gamma=0.01 g$, and $\gamma_{\phi}=0.01 g$, we obtain $D(X / 2)=2.2326, D(X / 2)=3.978$, and $D(X / 2)=3.812$, corresponding to error rates $P_{e}=0.012, P_{e}=3.47 \times 10^{-5}$, and $P_{e}=6.89 \times 10^{-5}$, respectively. For $g=10 \mathrm{MHz}$ [29], the docoherence rate/coherence time is about $100 \mathrm{kHz} / 10 \mu \mathrm{s}$. In the previous schemes $[17,18]$ for discrimination of chiral molecules by using difference of population, due to the effect of energy relaxation, long lifetime of higher molecule levels about $\tau \sim 200 \mu \mathrm{s}-$ $300 \mu \mathrm{s}(\gamma \sim 3.3 \mathrm{kHz}-5 \mathrm{kHz})$ is required to get acceptable successful probabilities $(\sim 0.99)$. However, it is hard for a higher molecule level to possess such long lifetime for various types of molecules. Moreover, the effect of dephasing is also not negligible in practice. When the dephasing is taken into account, the successful probability of schemes $[17,18]$ may be further reduced. In the current scheme, since the molecule level is almost restricted in the ground state, the evolution is insensitive to both energy relaxation and dephasing, thus greatly relax the requirement of coherence time. Although cavity decay caused more influence compared the other two factors, the 
error rate $P_{e}=0.012$ is still acceptable to discriminate chiral molecule. In fact, errors caused by cavity decay can be further reduced. When cavity decay is considered, the evolution can be analyzed with superoperators as $\rho(t+d t)=e^{\mathcal{L}[a] d t} \mathcal{U}(t, d t) \rho(t)$, with $\mathcal{U}(t, d t) \rho(t)=e^{-i H_{e}(t) d t} \rho(t) e^{i H_{e}(t) d t}$ [30]. Noticing the result

$$
\begin{aligned}
& e^{\mathcal{L}[a] d t}|\alpha\rangle\langle\alpha|=(1+\mathcal{L}[a] d t)| \alpha\rangle\langle\alpha|+\mathcal{O}\left(d t^{2}\right) \\
& =|\alpha(1-\kappa d t / 2)\rangle\langle\alpha(1-\kappa d t / 2)|+\mathcal{O}\left(d t^{2}\right)
\end{aligned}
$$

the evolution can be calculated as

$$
\begin{array}{r}
\bar{\rho}(T)=| \pm \bar{\alpha}(T)\rangle_{\mathcal{c}}\langle \pm \bar{\alpha}(T)|\otimes| 1\rangle_{e}\langle 1|, \\
\bar{\alpha}(T)=e^{i \varphi(T)-\kappa T / 2} \int_{0}^{T} i \Omega_{e}(t) e^{\kappa t / 2-i \varphi(t)} d t .
\end{array}
$$

Although cavity decay is hard to avoid, the decay rate may be predetermined via experiments. When decay rate is known, we can make correction of pulse as $\tilde{\Omega}(t) \rightarrow \tilde{\Omega}(t) \exp [\kappa(T-$ t) /2] to make $\bar{\alpha}(T)=\alpha(T)$. As shown in Fig. 3(e), $D(X / 2)$ versus decoherence rate $\left(\gamma=\gamma_{\phi}=\kappa\right)$ is plotted with corrected pulses and original pulses. The result indicate that when the corrected pulses are applied, the discrimination is improved a lot compared with that using original pulses. For $\gamma=\gamma_{\phi}=$ $\kappa=0.01 g$, we still have $D(X / 2)=2.575$, corresponding to error rate $P_{e}=0.005$. In addition, we can also reduce error rates by measuring cavity fields at a time $T^{\prime}$ before $T$ since the maximal value of $D(X / 2)$ does not appear at $T$ when decoherence is included. As an example, we plot $D(X / 2)$ versus $t / T$ with $\gamma=\gamma_{\phi}=\kappa=0.01 \mathrm{~g}$ in Fig. 3(f), where we can find that with the correction, the maximum $D(X / 2)=3.367\left(P_{e}=3.8 \times 10^{-4}\right)$ appears at $T^{\prime}=165 / g$, and without the correction, the maximum $D(X / 2)=2.639\left(P_{e}=0.004\right)$ appears at $T^{\prime}=183 / g$. By measuring cavity fields at $t=T^{\prime}$, the successful probabilities can be significantly improved. Furthermore, recent schemes for intensifying coupling strengths [31] or improving quality factors [32] make it possible to enhance the ratio $g / \kappa$ in a cavity. These techniques can make the scheme works more efficiently in a real scenario.

As an example for the implementation of the scheme, the rotational states $\left|0_{00}\right\rangle,\left|1_{11}\right\rangle$ and $\left|1_{10}\right\rangle$ of 1,2-propanediol molecules with $\left|J_{k_{-1}, k_{1}}\right\rangle$ being the rotational state with quantum numbers of the limiting prolate and oblate symmetric top $k_{-1}$ and $k_{1}$, can be considered as levels $|1\rangle,|2\rangle$ and $|3\rangle$, respectively [11]. The transition frequencies between these three states are $\omega_{12}=11363 \mathrm{MHz}, \omega_{13}=12212 \mathrm{MHz}$, and $\omega_{2,3}=849 \mathrm{MHz}$ in this model, and the frequencies of pulses $\Omega(t)$ and $\tilde{\Omega}(t)$ should be $\omega=11563 \mathrm{MHz}$ and $\tilde{\omega}=12202 \mathrm{MHz}$. In addition, the frequency of the cavity mode is $v=639 \mathrm{MHz}$.

In conclusion, we have proposed an effective scheme to discriminate chiral molecule with the help of a cavity. We showed that enantiomers possessing different handedness produce coherence states of cavity mode with contrary-sign displacements $\pm \alpha(T)$, consequently information about the handedness can be read out by homodyne measurement [25-28] with very slight error rates. The scheme does not require very strong coherence state. When $|\alpha(T)|=2$ (average photon number $\bar{n}=4$ ), the error rate is only $3.16712 \times 10^{-5}$ in theory. Moreover, the scheme is robust against deviations of displacements caused by systematic errors and random noise. Because when $|\alpha(T)|$ is big enough, deviations of displacements only induce tiny change of error rates. Compared with the scheme $[17,18]$ based on difference of populations, the scheme provide a faulttolerance approach for the discrimination since populations of molecules are more sensitive to errors of pulses. Furthermore, as molecules are almost restricted in the ground states, the scheme also holds better robustness against energy relaxation and dephasing of molecules. With synthetical consideration of energy relaxation, dephasing and cavity decay, the scheme can still produce acceptable successful probability with coherence time only about $10 \mu \mathrm{s}$. Therefore, the scheme may be useful to realize accurate discrimination of chiral molecule in the presence of experimental imperfections.

Funding. National Natural Science Foundation of China (11805036)

Disclosures. The authors declare no conflicts of interest.

\section{REFERENCES}

1. K. T. Barrett, A. J. Metrano, P. R. Rablen, and S. J. Miller, Nature 509, 71 (2014).

2. J. Gal, Chirality 24, 959 (2012).

3. C. L. Amorim, I. S. Moreira, A. R. Ribeiro, L. H. Santos, C. DelerueMatos, M. E. Tiritan, and P. M. Castro, Int. Biodeter. Biodegr. 115, 277 (2016).

4. W. S. Knowles, Angew. Chem. Int. Ed. Engl. 41, 1998 (2002).

5. Chiral Separation Methods for Pharmaceutical and Biotechnological Products, edited by S. Ahuja (John Wiley \& Sons, New York, 2011).

6. Y. Fujimura, L. Gonzĺez, K. Hoki, J. Manz, and Y. Ohtsuki, Chem. Phys. Lett. 306, 1 (1999).

7. K. Hoki, D. Kröner, and J. Manz, Chem. Phys. 267, 59 (2001).

8. L. González, D. Kröner, and I. R. Solá, J. Chem. Phys. 115, 2519 (2001).

9. K. Hoki, L. González, and Y. Fujimura, J. Chem. Phys. 116, 8799 (2002).

10. A. Jacob and K. Hornberger, J. Chem. Phys. 137, 044313 (2012).

11. D. Patterson, M. Schnell, and J. M. Doyle, Nature 497, 475 (2013).

12. M. Shapiro, E. Frishman, and P. Brumer, Phys. Rev. Lett. 84, 1669 (2000).

13. P. Král and M. Shapiro, Phys. Rev. Lett. 87, 183002 (2001).

14. P. Král, I. Thanopulos, M. Shapiro, and D. Cohen, Phys. Rev. Lett. 90, 033001 (2003).

15. Y. Li and C. Bruder, Phys. Rev. A 77, 015403 (2008).

16. N. V. Vitanov and M. Drewsen, Phys. Rev. Lett. 122, 173202 (2019).

17. J. L. Wu, Y. Wang, J. Song, Y. Xia, S. L. Su, and Y. Y. Jiang, Phys. Rev. A 100, 043413 (2019).

18. J. L. Wu, Y. Wang, J. X. Han, C. Wang, S. L. Su, Y. Xia, Y. Jiang, and J. Song, Phys. Rev. Applied 13, 044021 (2020).

19. X. Chen, I. Lizuain, A. Ruschhaupt, D. Guéry-Odelin, and J. G. Muga, Phys. Rev. Lett. 105, 123003 (2010).

20. A. del Campo, Phys. Rev. Lett. 111, 100502 (2013).

21. A. del Campo, M. M. Rams, and W. H. Zurek, Phys. Rev. Lett. 109, 115703 (2012).

22. S. Masuda and K. Nakamura, Phys. Rev. A 84, 043434 (2011).

23. D. F. V. James and J. Jerke, Can. J. Phys. 85, 625 (2007).

24. A. Luis, J. Phys. A 34, 7677 (2001).

25. J. M. Torres, J. Z. Bernád, and G. Alber, Phys. Rev. A 90, 012304 (2014).

26. K. Nemoto and W. J. Munro, Phys. Rev. Lett. 93, 250502 (2004).

27. S. D. Barrett, P. Kok, K. Nemoto, R. G. Beausoleil, W. J. Munro, and T. P. Spiller, Phys. Rev. A 71, 060302(R) (2005).

28. C. A. González-Gutiérrez and J. M. Torres, Phys. Rev. A 99, 023854 (2019).

29. W. Lu, Y. Zhao, and P. F. Barker, Phys. Rev. A 76, 013417 (2007).

30. S. B. Zheng, Phys. Rev. A 91, 052117 (2015).

31. W. Qin, A. Miranowicz, P. B. Li, X. Y. Lü, J. Q. You, and F. Nori, Phys. Rev. Lett. 120, 093601 (2018).

32. N. Acharyya and G. Kozyreff, Phys. Rev. Applied 12, 014060 (2019). 


\section{REFERENCES}

1. K. T. Barrett, A. J. Metrano, P. R. Rablen, and S. J. Miller, Spontaneous transfer of chirality in an atropisomerically enriched two-axis system, Nature 509, 71 (2014).

2. J. Gal, The discovery of stereoselectivity at biological receptors: Arnaldo piutti and the taste of the asparagine enantiomers-history and analysis on the 125th anniversary, Chirality 24, 959 (2012).

3. C. L. Amorim, I. S. Moreira, A. R. Ribeiro, L. H. Santos, C. DelerueMatos, M. E. Tiritan, and P. M. Castro, Treatment of a simulated wastewater amended with a chiral pharmaceuticals mixture by an aerobic granular sludge sequencing batch reactor, Int. Biodeter. Biodegr. 115, 277 (2016).

4. W. S. Knowles, Asymmetric Hydrogenations (Nobel Lecture), Angew. Chem. Int. Ed. Engl. 41, 1998 (2002).

5. Chiral Separation Methods for Pharmaceutical and Biotechnological Products, edited by S. Ahuja (John Wiley \& Sons, New York, 2011).

6. Y. Fujimura, L. Gonzlez, K. Hoki, J. Manz, and Y. Ohtsuki, Selective preparation of enantiomers by laser pulses: quantum model simulation for H2POSH, Chem. Phys. Lett. 306, 1 (1999).

7. K. Hoki, D. Kröner, and J. Manz, Selective preparation of enantiomers from a racemate by laser pulses: model simulation for oriented atropisomers with coupled rotations and torsions, Chem. Phys. 267, 59 (2001).

8. L. González, D. Kröner, and I. R. Solá, Separation of enantiomers by ultraviolet laser pulses in H2POSH: $\pi$ pulses versus adiabatic transitions, J. Chem. Phys. 115, 2519 (2001).

9. K. Hoki, L. González, and Y. Fujimura, Quantum control of molecular handedness in a randomly oriented racemic mixture using three polarization components of electric fields, J. Chem. Phys. 116, 8799 (2002).

10. A. Jacob and K. Hornberger, Effect of molecular rotation on enantioseparation, J. Chem. Phys. 137, 044313 (2012).

11. D. Patterson, M. Schnell, and J. M. Doyle, Enantiomer-specific detection of chiral molecules via microwave spectroscopy, Nature 497, 475-477 (2013).

12. M. Shapiro, E. Frishman, and P. Brumer, Highly efficient detection and separation of chiral molecules through shortcuts to adiabaticity, Phys. Rev. Lett. 84, 1669 (2000).

13. P. Král and M. Shapiro, Cyclic population transfer in quantum systems with broken symmetry, Phys. Rev. Lett. 87, 183002 (2001).

14. P. Král, I. Thanopulos, M. Shapiro, and D. Cohen, Two-step enantioselective optical switch, Phys. Rev. Lett. 90, 033001 (2003).

15. Y. Li and C. Bruder, Dynamic method to distinguish between left- and right-handed chiral molecules, Phys. Rev. A 77, 015403 (2008).

16. N. V. Vitanov and M. Drewsen, Highly efficient detection and separation of chiral molecules through shortcuts to adiabaticity, Phys. Rev. Lett. 122, 173202 (2019).

17. J. L. Wu, Y. Wang, J. Song, Y. Xia, S. L. Su, and Y. Y. Jiang, Robust and highly efficient discrimination of chiral molecules through threemode parallel paths, Phys. Rev. A 100, 043413 (2019).

18. J. L. Wu, Y. Wang, J. X. Han, C. Wang, S. L. Su, Y. Xia, Y. Jiang, and J. Song, Two-path interference for enantiomer-selective state transfer of chiral molecules, Phys. Rev. Applied 13, 044021 (2020).

19. X. Chen, I. Lizuain, A. Ruschhaupt, D. Guéry-Odelin, and J. G. Muga, Shortcut to adiabatic passage in two- and three-level atoms, Phys. Rev. Lett. 105, 123003 (2010).

20. A. del Campo, Shortcuts to adiabaticity by counterdiabatic driving, Phys. Rev. Lett. 111, 100502 (2013).

21. A. del Campo, M. M. Rams, and W. H. Zurek, Assisted finite-rate adiabatic passage across a quantum critical point: exact solution for the quantum Ising model, Phys. Rev. Lett. 109, 115703 (2012).

22. S. Masuda and K. Nakamura, Acceleration of adiabatic quantum dynamics in electromagnetic fields, Phys. Rev. A 84, 043434 (2011).

23. D. F. V. James and J. Jerke, Effective Hamiltonian theory and its applications in quantum information, Can. J. Phys. 85, 625 (2007).

24. A. Luis, Quantum mechanics as a geometric phase: phase-space interferometers, J. Phys. A 34, 7677 (2001).

25. J. M. Torres, J. Z. Bernád, and G. Alber, Quantum teleportation and entanglement swapping of matter qubits with coherent multiphoton states, Phys. Rev. A 90, 012304 (2014).

26. K. Nemoto and W. J. Munro, Nearly deterministic linear optical controlled-NOT gate, Phys. Rev. Lett. 93, 250502 (2004).

27. S. D. Barrett, P. Kok, K. Nemoto, R. G. Beausoleil, W. J. Munro, and T. P. Spiller, Symmetry analyzer for nondestructive Bell-state detection using weak nonlinearities, Phys. Rev. A 71, 060302(R) (2005).

28. C. A. González-Gutiérrez and J. M. Torres, Atomic Bell measurement via two-photon interactions, Phys. Rev. A 99, 023854 (2019).

29. W. Lu, Y. Zhao, and P. F. Barker, Cooling molecules in optical cavities, Phys. Rev. A 76, 013417 (2007).

30. S. B. Zheng, Geometric phase for a driven quantum field subject to decoherence, Phys. Rev. A 91, 052117 (2015).

31. W. Qin, A. Miranowicz, P. B. Li, X. Y. Lü, J. Q. You, and F. Nori, Exponentially Enhanced Light-Matter Interaction, Cooperativities, and Steady-State Entanglement Using Parametric Amplification, Phys. Rev. Lett. 120, 093601 (2018).

32. N. Acharyya and G. Kozyreff, Large $Q$ factor with very small whispering-gallery-mode resonator, Phys. Rev. Applied 12, 014060 (2019). 